\title{
Computational strategy for studying structural change of tritium-substituted macromolecules by a beta decay to helium-3
}

\author{
Susumu Fujiwara ${ }^{1 *}$, Hiroaki Nakamura ${ }^{2,3}$, Haolun $\mathrm{Li}^{1}$, Hisanori Miyanishi ${ }^{3}$, \\ Tomoko Mizuguchi $^{1}$, Takuo Yasunaga ${ }^{4}$, Takao Otsuka ${ }^{5}$, Yuji Hatano ${ }^{6}$, Shinji Saito ${ }^{7}$ \\ ${ }^{1}$ Faculty of Materials Science and Engineering, Kyoto Institute of Technology \\ ${ }^{2}$ Department of Helical Plasma Research, National Institute for Fusion Science \\ ${ }^{3}$ Department of Energy Engineering and Science, Nagoya University \\ ${ }^{4}$ Department of Bioscience and Bioinformatics, Kyushu Institute of Technology \\ ${ }^{5}$ Quantitative Biology Center, RIKEN \\ ${ }^{6}$ Organization for Promotion of Research, University of Toyama \\ ${ }^{7}$ Institute for Molecular Science \\ ${ }^{\star}$ fujiwara@kit.ac.jp
}

Received: December 4, 2018; Accepted: January 23, 2019; Published: March 15, 2019

\begin{abstract}
We propose a computational strategy for investigating structural change of tritiumsubstituted macromolecules. Effects of radiation on macromolecules such as polymeric materials and DNA are classified into three categories: (1) direct action, (2) indirect action, and (3) decay effect. In this study, we focus on the decay effect exclusively. After a beta decay of substituted tritium in macromolecules to helium-3, the generated inert helium-3 is assumed to be deleted quickly. To get an insight into the decay effect to the damage of macromolecules, we perform molecular dynamics simulations of tritium-deleted macromolecules and analyze their structural change. Preliminary simulation results of decay effect on polymeric materials and DNA are presented.
\end{abstract}

Keywords: Molecular dynamics simulation, Structural change, Macromolecule, Polymeric materials, DNA, Decay effect

\section{Introduction}

Treatment of a large amount of contaminated water including tritium $(\mathrm{T})$ has been a problem in nuclear accident at Fukushima in 2011. Since the range of beta rays emitted from tritium 


\section{Journal of Advanced Simulation in Science and Engineering}

is short, external exposure is rarely a problem and protection against internal exposure is important [1]. Particularly, DNA damage caused by beta rays and DNA damage in the case where substituted tritium decays to helium-3 are regarded as problems. However, the molecular mechanism of the DNA damage is still an unsolved problem. Effects of radiation on macromolecules such as polymeric materials and DNA are classified into three categories: (1) direct action in which the radiation hits directly to the macromolecules and disrupts their molecular structures, (2) indirect action in which radiation-induced free radicals and active oxygen strike and disrupt macromolecules, and (3) decay effect in which chemical bonds break by a beta decay of substituted tritium in the macromolecules to helium-3. Although there have been numerous experimental and computer simulation studies on the DNA damage through direct and indirect action, any studies have not been conducted on the DNA damage through decay effect because of the difficulties in extracting the decay effect only. Therefore, we aim to predict the structural change of tritium-substituted macromolecules such as polymeric materials and DNA by a beta decay to helium-3 using molecular dynamics (MD) simulations.

\section{Computational strategy}

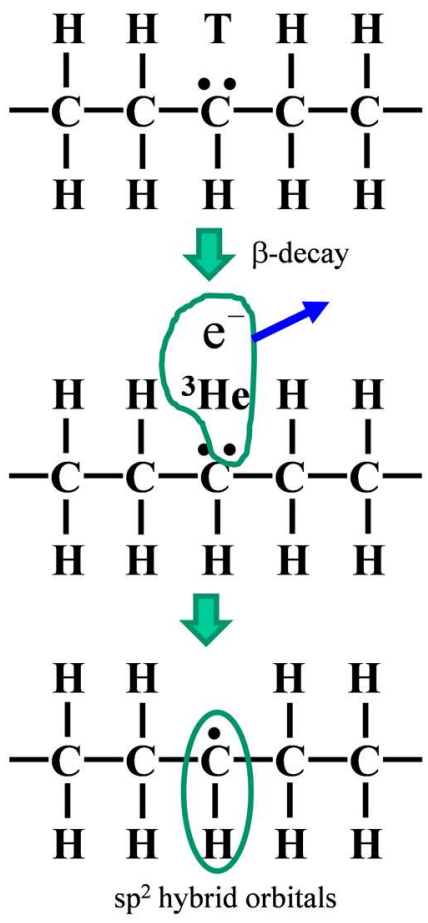

Figure 1: Treatment after a $\beta$-decay of substituted tritium in polyethylene to helium-3. Since helium-3 is inert, the ${ }^{3} \mathrm{He}-\mathrm{C}$ bond breaks, and after that the original -CHT- changes to $-\mathrm{CH}-$, which is treated as an $\mathrm{sp}^{2}$ hybrid orbital. 


\section{Journal of Advanced Simulation in Science and Engineering}

Tritium decays to helium-3 with emissions of beta-electron and an antineutrino with a half-life of 12.323 year [2]. The energy of the emitted beta-electrons is widely distributed; the maximum and average energies are $18.6 \mathrm{keV}$ and $5.7 \mathrm{keV}$, respectively. Since helium-3 is inert, chemical bonds of helium-3 break after a beta decay of substituted tritium in the macromolecules to helium-3, e.g., -CHT- changes to -CH- (Fig. 1). In this study, the generated helium-3 is assumed to be deleted quickly and we delete a certain amount of substituted tritium in the macromolecules to get an insight into the decay effect to the damage of macromolecules. To this end, we perform MD simulations of tritium-deleted macromolecules and analyze their structural change.

\section{Preliminary simulation results}

In this section, we show our preliminary simulation results of decay effects on polymeric materials and DNA.

\subsection{Decay effect on polymeric materials}

We consider a folded-chain crystal of polyethylene as polymeric materials. The computational model is similar to that used in Refs.[3,4]. The model polyethylene chain consists of $2998 \mathrm{CH}_{2}$ groups with 2 terminal $\mathrm{CH}_{3}$ groups which are all treated as united atoms. The mass of $\mathrm{CH}_{2}$ and $\mathrm{CH}_{3}$ groups are 14 and $15 \mathrm{~g} / \mathrm{mol}$, respectively. The united atoms interact via bonded potentials (bond-stretching, bond-bending and torsional potentials) and a non-bonded potential (12-6 Lennard-Jones potential). The atomic force field used here is the DREIDING potential [5]. We use the velocity Verlet algorithm and apply the Nosé-Hoover method in order to keep the temperature of the system constant. The integration time step is $1 \mathrm{fs}$. The total linear momentum and angular momentum are set to be zero in order to cancel overall translation and rotation of a polyethylene chain. The Large-scale Atomic/Molecular Massively Parallel Simulator (LAMMPS) is used for all calculations [6]. The MD simulations are carried out as follows. At first, we prepare the folded orientationally-ordered structure of a single polyethylene chain at $300 \mathrm{~K}$ by cooling from a random configuration at $800 \mathrm{~K}$. Next, we delete a certain amount of $\mathrm{H}$, which is regarded as substituted tritium, from a polyethylene chain randomly. The ratio of the number of deleted $\mathrm{H}$ to the number of $\mathrm{C}, f_{\mathrm{H}}$, is set as $f_{\mathrm{H}}=0,0.001,0.01$, and 0.1 . The tritium-deleted polyethylene is then heated to $800 \mathrm{~K}$.

Figure 2 shows the structural change of the tritium-deleted polyethylene with $f_{\mathrm{H}}=0.01$ during heating. It is found from this figure that the ordered structure becomes disordered between $550 \mathrm{~K}$ and $700 \mathrm{~K}$. In order to investigate the structural change of the folded orientationally-ordered structure in detail, we calculate the global orientational order parameter $P_{2}$, which 
(a)

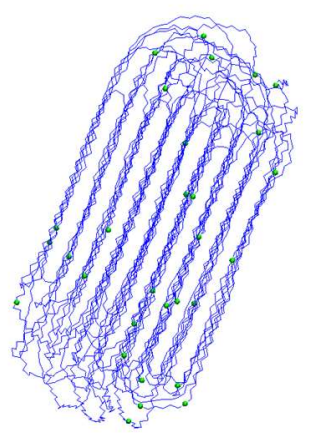

(b)

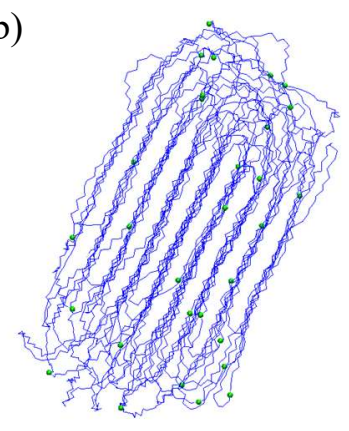

(c)

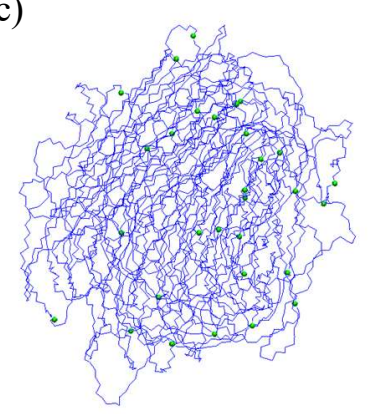

Figure 2: Structural change of the tritium-deleted polyethylene with $f_{\mathrm{H}}=0.01$ during heating (a) at $300 \mathrm{~K}$, (b) at $550 \mathrm{~K}$, and (c) at $700 \mathrm{~K}$. Green balls represent $\mathrm{CH}$ groups.

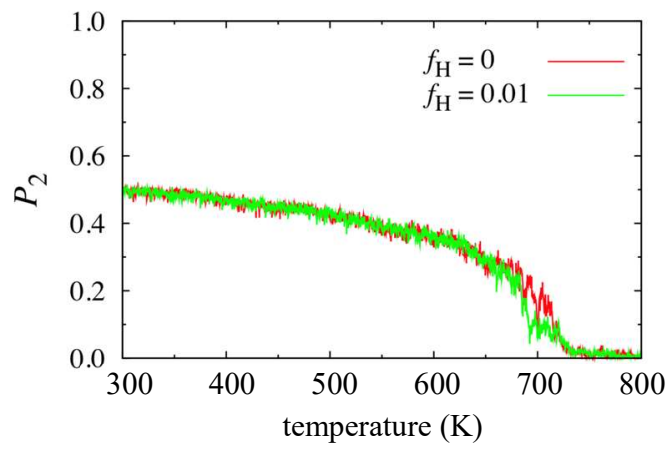

Figure 3: The global orientational order parameter $P_{2}$ versus temperature during heating for $f_{\mathrm{H}}=0$ (red) and $f_{\mathrm{H}}=0.01$ (green).

is defined by $P_{2}=\left\langle\left(3 \cos ^{2} \psi-1\right) / 2\right\rangle$, where, $\psi$ is the angle between two chord vectors $[3,4]$. The chord vector is the vector formed by connecting centers of two adjacent bonds along the chain. The brackets denote the average over all pairs of chord vectors. This parameter takes a value of 0 when chord vectors are randomly oriented and takes a value of 1 when all chord vectors are parallel. We show, in Fig. 3 , the global orientational order parameter $P_{2}$ as a function of temperature. This figure indicates that the transition temperature from the ordered structure to the disordered structure decreases as $f_{\mathrm{H}}$ increases.

\subsection{Decay effect on DNA}

We here consider a part of human telomere sequence as a DNA. The used force field is a modified CHARMM36 [7]. The MD calculations are performed by NAMD software [8]. In this study, we replace the hydrogen of Guanine with two ${ }^{3} \mathrm{He}$ atoms. Figure 4 shows our preliminary MD simulation results at $310 \mathrm{~K}$ in the case of $N=0$ and $N=8$, where $N$ is the number 


\section{Journal of Advanced Simulation in Science and Engineering}

of bases in which hydrogen in DNA is replaced by ${ }^{3} \mathrm{He}$. In the case of $N=8$, we can see the destruction of hydrogen bond clearly.

\section{Summary and future work}

We developed the simulation method to study the DNA damage and the damage of polymeric materials through decay effect, and performed the MD simulation to predict the structural change of tritium-substituted macromolecules such as DNA and polymeric materials by a beta decay to helium-3. Our preliminary simulation studies indicate that the ordered structure of tritium-deleted macromolecules is easier to break compared to the original macromolecules.

For future work, we will make a detailed analysis of the structural change such as the analysis of the conformation and the root mean square deviation. We will also perform the ReaxFF (reactive force field) MD simulations [9] to deal with the formation and breakage of the chemical bonds.
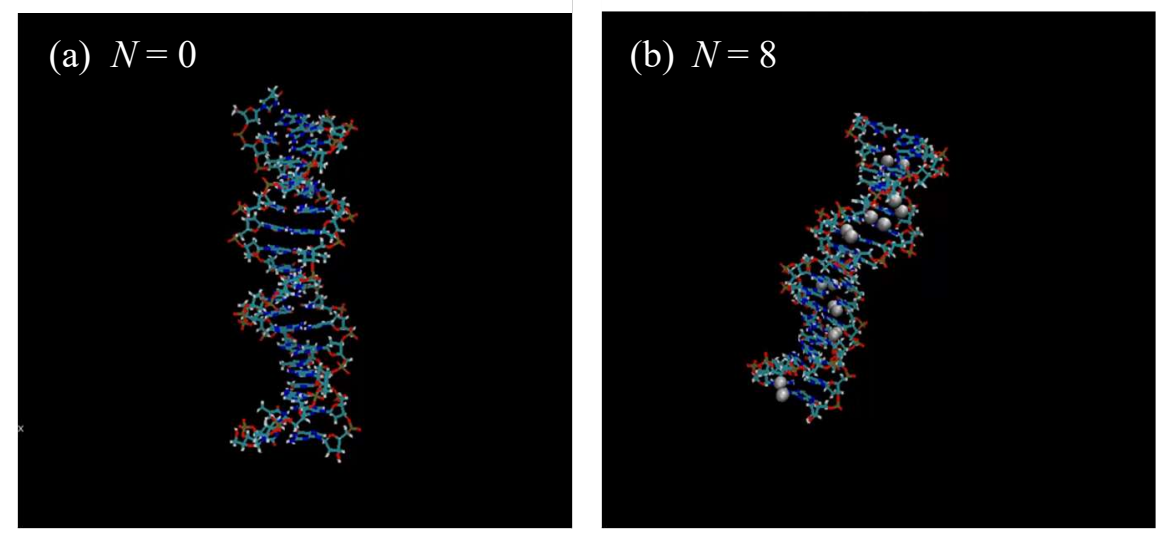

Figure 4: Snapshots of a part of human telomere sequence at 310K (a) for $N=0$ and (b) for $N=8$. Gray balls in (b) represent healium-3. Note that water molecules are not shown for clarity.

\section{Acknowledgement}

This work was partially supported by the Joint Studies Program (2017-2019) of the Institute for Molecular Science, the Joint Research by the National Institutes of Natural Sciences (NINS), and was partially performed with the support and under the auspices of the National Institute for Fusion Science (NIFS) Collaboration Research program (NIFS17KNTS050).

\section{References}

[1] Tritium: Fuel of Fusion Reactors, edited by T. Tanabe (Springer Japan, 2017). 


\section{Journal of Advanced Simulation in Science and Engineering}

[2] P. C. Souers, Hydrogen Properties for Fusion Energy (University of California Press, California, 1986).

[3] S. Fujiwara, T. Sato: Molecular dynamics simulations of structural formation of a single polymer chain: Bond-orientational order and conformational defects, J. Chem. Phys., 107:2 (1997), 613-622.

[4] S. Fujiwara, T. Sato: Structure formation of a single polymer chain. I: Growth of trans domains, J. Chem. Phys., 114:14 (2001), 6455-6463.

[5] S. L. Mayo, B. D. Olafson, W. A. Goddard III: DREIDING: A Generic Force Field for Molecular Simulations, J. Phys. Chem., 94:26 (1990), 8897-8909.

[6] S. Plimpton: Fast Parallel Algorithms for Short-Range Molecular Dynamics, J. Comput. Phys., 117:1 (1995), 1-19.

[7] J. Huang, A. D. MacKerell Jr.: CHARMM36 all-atom additive protein force field: validation based on comparison to NMR data, J. Comput. Chem., 34:25 (2013), 2135-2145.

[8] J. C. Phillips, R. Braun, W. Braun, J. Gumbart, E. Tajkhorshid, E. Villa, C. Chipot, R. D. Skeel, L. Kale, K. Schulten: Scalable molecular dynamics with NAMD, J. Comput. Chem., 26:16 (2005), 1781-1802.

[9] A. C. T. van Duin, S. Dasgupta, F. Lorant, W. A. Goddard III : ReaxFF: A Reactive Force Field for Hydrocarbons, J. Phys. Chem. A, 105:41 (2001), 9396-9409. 